\title{
A numerical method to generate optimal load paths in plain and reinforced concrete structures
}

\author{
Matteo Bruggi \\ Department of Civil and Environmental Engineering, Politecnico di Milano, I20133, Milano, Italy
}

\begin{abstract}
A numerical method based on topology optimization is proposed to generate optimal strutonly models for structures made of plain concrete and optimal strut-and-tie models for concrete structures where fixed regions of reinforcement are prescribed. Assuming concrete as a hyperelastic material carrying only compression, both the inherently non linear equilibrium equation and the energy-based topology optimization problem are solved within the same minimization procedure. Numerical simulations investigate load paths within the two-dimensional domain in case of conventional rebar cages. A stress diffusion problem is considered as well.

Keywords: topology optimization, strut-and-tie models, reinforced concrete structures, plain concrete structures, energy-based methods.
\end{abstract}

\section{Introduction}

Strut-and-tie models (STMs) are of primary importance when designing reinforced concrete members. This detailing approach investigates truss-like structures to define statically admissible load paths that connect the load application points to the ground constraints, see e.g. [1]. It was firstly introduced to cope with the transversal reinforcement for shear design of beams [2,3] and then extended to the so-called D-regions of reinforced concrete structures, where the Bernoulli

Email address: matteo.bruggi@polimi.it (Matteo Bruggi) 
hypothesis does not hold due to any discontinuity in the geometry of the specimen or in the load pattern [4]. A complex strain/stress state arises in these regions, meaning that the conventional assumption about cross sections remaining plane after bending falls. This implies that standard design methods based on the beam theory can not be applied. According to the plasticity theorem, any statically admissible truss-like structure that does not violate the yield criteria has a loadcarrying capacity that is a lower-bound of the ultimate strength of the specimen. This supports the adoption of strut-and-tie modeling to define statically admissible load paths for a proper detailing of the D-regions.

Methods of structural optimization have been used to generate suitable strut-and-tie models among the number of truss-like structures that can be defined within a certain domain. The ground structure approach was firstly adopted to select minimum energy or minimum weight strut-and-tie models addressing grids of discrete trusses, see e.g. [5-8]. Afterwards, topology optimization was introduced to generate optimal truss-like layouts adopting the same objective functions but resorting to the finite element modeling for plane problems. Evolutionary structural optimization was used in the pioneering approaches proposed in [9-13], whereas the distribution of solid isotropic material according to the SIMP model for stiffness penalization [14] was adopted e.g. in [15-20]. The latter approach has also been used for the conceptual design of optimal layouts of steel reinforcement dropping the conventional strut-and-tie approach and moving towards more general problems of topology optimization for reinforced concrete structures, involving nonsymmetric strength constraints, see e.g. [21-23], damage mechanics [24, 25], elastoplasticity, see [26]. Remaining in the field of strut-and-tie modeling, an alternative approach combining truss design and topology optimization has been recently presented in $[27,28]$ to look for load paths that are not only statically admissible but also consistent with the stress flow in the concrete element.

To ensure appropriate safety standards, designers are generally required by technical codes to neglect the limited tensile strength of concrete when detailing reinforced concrete beam sections at 
the ultimate limit state. Indeed, strut-and-tie modeling stands on the assumption that concrete carries compressive stresses only, whereas steel is used as a reinforcement to carry any tensile stress. According to technical rules, best STMs can be selected through energy-based criteria [29]. These prescriptions explain the strong interest of the community of designers towards the adoption of simple numerical methods exploiting topology optimization to distribute linear elastic isotropic material and achieve truss-like layouts as preferred strut-and-tie load paths, see [30]. Assessment of the achieved results and detailing of each member making the optimal STM can be performed subsequently through ad hoc tools, see e.g. [31].

Unfortunately, the linear elastic approach fails when addressing the achievement of energybased load paths in structures where the layout of the reinforcement is already prescribed (due to the adoption of a fixed rebar cage) or when copying with the optimal design of plain concrete members (with no rebar allowed except for the secondary reinforcement intended for ductility and durability requirements only). In these cases, conventional methods of topology optimization should be modified to avoid the arising of any tensile-stressed member within the concrete domain. This can be conveniently pursued by implementing methods of energy-based topology optimization for compression-only materials.

Alternative formulations have been proposed in the last decades to address tension-only or compression-only materials in topology optimization. Most of them resort to non-linear modeling, see e.g. [32], or to re-modeling theories and material replacement strategies that distribute the unilateral material depending on the directions of the stress flows computed in the design domain, see e.g. [33, 17, 34]. A simplified stress-based approach has been presented in [35] that implements a smooth approximation of the unilateral condition through the formulation of a suitable version of the Drucker-Prager strength criterion. Due to the inherent isotropic modeling, this approach can not be straightforwardly applied to structures governed by any complex biaxial stress state. In this case, a full no-tension/no-compression modeling of the constitutive behavior of the material 
should be implemented.

Hyper-elasticity of unilateral materials allows solving the inherently nonlinear equilibrium through the minimization of the strain energy, see in particular [36]. The energy-based approach presented in [37] is herein exploited to address the generation of optimal STMs through a topology optimization problem that copes with concrete as a compression-only material. An equivalent orthotropic medium is defined, exhibiting negligible stiffness for any direction along which a tensile principal stress must be prevented. Two sets of density unknowns are needed to control the stiffness of the equivalent composite along its symmetry axes, which should be oriented along the principal stress directions of the no-tension body. The topology optimization problem adopts the weight as objective function, whereas a global constraint is prescribed on the overall compliance of the specimen (made of plain concrete or concrete reinforced by a fixed rebar cage). Its solution is performed adopting an established method of sequential convex programming, the Method of Moving Asymptotes MMA [38]. The compression-only behavior is efficiently enforced through an ad hoc penalization of the energy terms related to any tensile stress arising during the optimization.

The layout of the paper is as follows. Section 2 recalls the rationale used to model a compressiononly solid as an equivalent orthotropic medium, whereas Section 3 introduces the energy-based topology optimization problem that is used to derive optimal STMs addressing concrete as a compression-only material. Section 3.1 provides details on the implementation of the solving algorithm. Features of the achieved optimal STMs are discussed in Section 4, presenting numerical examples and providing comparison with optimal layouts found through the conventional approach with linear elastic modeling and equal behavior in tension and compression of the material. Section 4.1 is devoted to optimal strut-only models for structures made of plain concrete. Section 4.2 deals with optimal strut-and-tie models for concrete structures where fixed regions of reinforcement are prescribed. Section 5 resumes the main findings of the work, outlining future extensions of this research. 

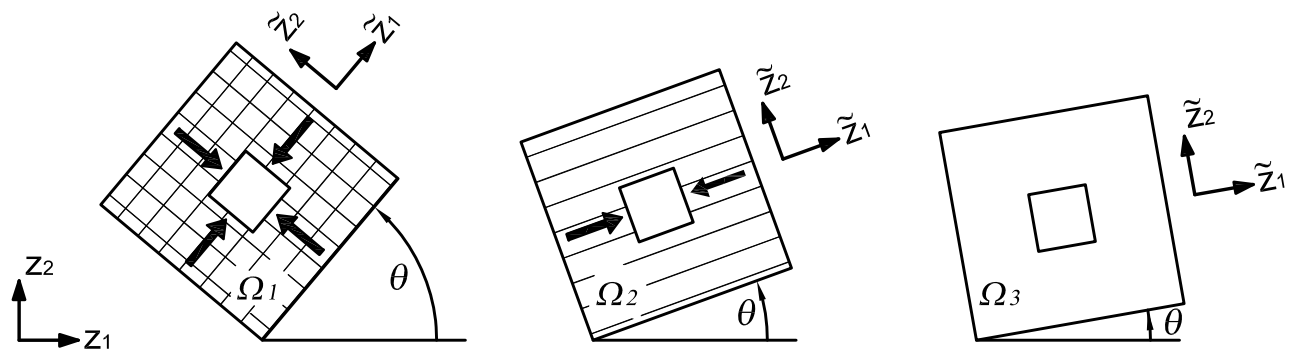

Figure 1: Material model in case of a compression-only material in the general reference of axes $z_{1}$ and $z_{2}$ in $\Omega$ : an orthotropic phase with symmetry axes $\widetilde{z}_{1}$ and $\widetilde{z}_{2}$ is oriented along the principal stress directions $z_{I}$ and $z_{I I}$. Directions where stiffness of the composite is not negligible are represented through bold lines.

\section{A material interpolation for concrete}

Young modulus $E$ and non-negative Poisson's ratio $\nu$ are herein assumed for a material that can sustain only compressive stresses, dealing with plane stress conditions. The principal axis $z_{I I I}$ is orthogonal to the reference plane whose coordinates are labeled $z_{1}$ and $z_{2}$. Let $\sigma_{I}, \sigma_{I I}$ and $\sigma_{I I I}=0$ be the principal stresses for the tensor $\sigma_{i j}(\chi)$, as computed at any point $\chi \in \Omega$, with $\sigma_{I} \leq \sigma_{I I}$

The domain $\Omega$ is divided into three sub-regions such that $\Omega=\Omega_{1} \cup \Omega_{2} \cup \Omega_{3}$ and:

$$
\begin{aligned}
& \Omega_{1}=\chi \in \Omega: \sigma_{I}<0, \sigma_{I I}<0, \\
& \Omega_{2}=\chi \in \Omega: \sigma_{I}<0, \sigma_{I I}=0, \\
& \Omega_{3}=\chi \in \Omega: \sigma_{I}=0 .
\end{aligned}
$$

In sub-region $\Omega_{1}$ the material is acted upon by biaxial compression and behaves as any conventional isotropic medium. In $\Omega_{2}$ the material is acted upon by uniaxial compression and behaves as an orthotropic medium. A fully elastic behavior is recovered along the compressive isostatic line, whereas some inelastic strain $\varepsilon^{c} \geq 0$ arises in the orthogonal direction. In sub-region $\Omega_{3}$ neither stress nor elastic strain is found and the solid behaves as a "void phase", meaning that any positive 
semi-definite inelastic strain is allowed.

An equivalent orthotropic material model has been formulated in [37] to describe the outlined behavior through the same closed-form expression at any point $\chi \in \Omega$. This may be done introducing two fields of density unknowns that allow specializing the behavior of the solid in each of the sub-regions $\Omega_{1}, \Omega_{2}$ and $\Omega_{3}$ of Eqn. (1), penalizing the stiffness of the orthotropic material along its symmetry axes $\widetilde{z}_{1}$ and $\widetilde{z}_{2}$. Principal stress directions $z_{I}$ and $z_{I I}$ and the symmetry axes $\widetilde{z}_{1}$ and $\widetilde{z}_{2}$ share the same orientation, as provided by the angle $\theta$, see Figure 1 . Referring to the elastic constants of the equivalent orthotropic medium, $\widetilde{E}_{1}, \widetilde{E}_{2}$ are the Young moduli of the material (along $\widetilde{z}_{1}$ and $\widetilde{z}_{2}$, respectively), $\widetilde{G}_{12}$ is the shear modulus and $\widetilde{\nu}_{12}, \widetilde{\nu}_{21}$ are the Poisson's ratios. It is recalled that the equality $\widetilde{\nu}_{i j} \widetilde{E}_{j}=\widetilde{\nu}_{j i} \widetilde{E}_{i}$ holds.

The design problem will be solved through a displacement-based finite element method, adopting four node bilinear elements. In view of the adoption of such a discretization, let denote as $x_{1 e}$ and $x_{2 e}$ the density unknowns that govern the behavior of the equivalent composite along its symmetry axes (i.e. the isostatic stress lines of the compression-only material) related to each element of the mesh. Following a generalization of the SIMP (Solid Isotropic Material with Penalization) $[14,39]$ used in topology optimization for isotropic materials, the elastic constants for the equivalent orthotropic material can be written as:

$$
\widetilde{E}_{i}=x_{i e}^{p} E, \quad \widetilde{G}_{i j}=x_{i e}^{p} x_{j e}^{p} \frac{E}{2(1+\nu)}, \quad \widetilde{\nu}_{i j}=x_{j e}^{p} \nu, \quad \text { for } i, j=1,2 .
$$

Reference is made to $[40,41]$ for the original development of the above formula within the framework of derivable optimality criteria methods for structural optimization. As usually done in conventional formulations for the topology optimization of isotropic materials the penalization parameter is assumed such that $p=3$, see in particular [30]. The interpolation in Eqn. (2) is especially conceived to provide vanishing stiffness along any direction along which the relevant density unknown, $x_{1 e}$ or $x_{2 e}$, takes its minimum value. In the general reference with axes $z_{1}$ and 
$z_{2}$, denoting by $\underline{\sigma}_{e}=\left[\sigma_{11} \sigma_{22} \sigma_{12}\right]$ the vector of the stress components for the $e$-th element and by $\underline{\varepsilon}_{e}=\left[\begin{array}{lll}\varepsilon_{11} & \varepsilon_{22} & 2 \varepsilon_{12}\end{array}\right]$ the corresponding strain components, the constitutive law for the equivalent orthotropic material reads:

$$
\underline{\sigma}_{e}=\mathbf{T}\left(\theta_{e}\right)^{-1} \mathbf{C}\left(x_{1 e}, x_{2 e}\right) \mathbf{T}\left(\theta_{e}\right)^{-t} \underline{\varepsilon}_{e},
$$

where $\mathbf{C}$ is the constitutive matrix written in the material reference with axes $\widetilde{z}_{1}$ and $\widetilde{z}_{2}$ and $\mathbf{T}$ is a transformation matrix, i.e.:

$$
\mathbf{C}=\left[\begin{array}{ccc}
\frac{\widetilde{E}_{1}}{1-\widetilde{\nu}_{12} \widetilde{\nu}_{21}} & \frac{\widetilde{\nu}_{12} \widetilde{E}_{1}}{1-\widetilde{\nu}_{12} \widetilde{\nu}_{21}} & 0 \\
\frac{\widetilde{\nu}_{21} \widetilde{E}_{2}}{1-\widetilde{\nu}_{12} \widetilde{\nu}_{21}} & \frac{\widetilde{E}_{2}}{1-\widetilde{\nu}_{12} \widetilde{\nu}_{21}} & 0 \\
0 & 0 & \widetilde{G}_{12}
\end{array}\right] \text { and } \mathbf{T}=\left[\begin{array}{ccc}
\cos ^{2} \theta_{e} & \sin ^{2} \theta_{e} & 2 \cos \theta_{e} \sin \theta_{e} \\
\sin ^{2} \theta_{e} & \cos ^{2} \theta_{e} & -2 \cos \theta_{e} \sin \theta_{e} \\
-\cos \theta_{e} \sin \theta_{e} & \cos \theta_{e} \sin \theta_{e} & \cos ^{2} \theta_{e}-\sin ^{2} \theta_{e}
\end{array}\right]
$$

The parameter $\theta_{e}$ provides the orientation of the principal stress directions $z_{I}$ and $z_{I I}$ (hence, of the symmetry axes $\widetilde{z}_{1}$ and $\widetilde{z}_{2}$ ) in the $e$-th element, with respect to the general axes $z_{1}$ and $z_{2}$.

Specializing $\mathbf{C}$ in terms of the extremal values of the variables $x_{\min } \leq x_{1 e} \leq 1$ and $x_{m i n} \leq x_{2 e} \leq 1$, one may straightforwardly check that Eqns. (3-4) recover the behavior found in each one of the three sub-regions $\Omega_{1}, \Omega_{2}$ and $\Omega_{3}$ of Eqn. (1).

\section{An energy-based approach to the automatic generation of strut-and-tie models}

A classical formulation of topology optimization looks for the distribution of linear elastic isotropic material that minimizes the structural compliance, i.e. the work of external loads at equilibrium, for a prescribed amount of available material. This is generally given as a percentage of the design domain, the so-called volume fraction $0<V_{f}<1$. Low values of $V_{f}$ allows for the achievement of stiff truss-like structures that can be interpreted as optimal strut-and-tie models used for the disposal of the primary reinforcement. The static theorem implies that an energy- 
based STM can be considered as a lower bound of the load-carrying capacity of an elastic-plastic structural member, if the statically admissible truss does not violate the yield criterion, see e.g. [4]. Concrete structures are provided with the required ductility through the disposal of the secondary reinforcement. Denoting by $x_{e}$ the element-wise constant density entering the conventional SIMP model in the $e$-th finite element, truss-like models can be alternatively generated resorting to the following discrete statement:

$$
\begin{cases}\min _{x_{e}} & \mathcal{W}=\sum_{e=1}^{N} x_{e} A_{e} \\ \text { s.t. } & \sum_{e=1}^{N} \mathbf{K}_{e}\left(x_{e}\right) \mathbf{U}=\mathbf{F}, \\ & \sum_{e=1}^{N} \mathbf{U}_{e}^{T} \mathbf{K}_{e}\left(x_{e}\right) \mathbf{U}_{e} \leq \alpha \mathcal{C}_{0}, \\ & x_{\min } \leq x_{e} \leq 1 .\end{cases}
$$

In the above equation, the objective function is the the weight $\mathcal{W}$, which is computed multiplying the element density $x_{e}$ for the relevant area $A_{e}$ over the $N$ elements in the mesh. Eqn. (5.2) enforces the discrete equilibrium for the linear elastic isotropic medium, being $\mathbf{K}_{e}$ the element stiffness matrix, $\mathbf{U}$ the global displacement vector and $\mathbf{F}$ the vector accounting for the applied external forces (no body load is considered). Eqn. (5.3) is a constraint on the overall stiffness that requires the overall compliance $\mathcal{C}$ to be lower than a prescribed limit $\alpha \mathcal{C}_{0}$, where $\mathcal{C}_{0}$ is the compliance found for the full domain made of virgin material $\left(x_{e}=1\right.$ everywhere) and $\alpha$ is a user-defined parameter. $\mathcal{C}$ is computed as the sum of $N$ contributions depending on the element displacement vectors $\mathbf{U}_{e}$. Dealing with one point load, this simply means formulating a constraint on the maximum displacement allowed at the loaded point along the load direction, see in particular [11]. Eqn. (5.4) introduces a lower bound against singularity of the stiffness matrix due to "void" regions. 
Following [37], the equilibrium of a hyper-elastic compression-only solid may be solved distributing suitable phases of the orthotropic material defined in Section 2 in the sub-regions $\Omega_{1}, \Omega_{2}$ and $\Omega_{3}$ such that the elastic strain energy stored in the unilateral medium is minimized, see also [36]. Endowing the original formulation with a weight constraint, a limited amount of material is made available instead of the full domain. The topology optimization problem can be straightforwardly solved through the same minimization procedure, without resorting to any incremental approach. Truss-like models involving a compression-only material can be alternatively formulated minimizing the structural weight subject to a compliance constraint, which means enforcing a prescribed stiffness to the optimal design. Eqn. (5) is modified as:

$$
\begin{cases}\min _{x_{1 e}, x_{2 e}} & \mathcal{W}=\sum_{e=1}^{N}\left(x_{1 e}+x_{2 e}-x_{1 e} x_{2 e}\right) A_{e} \\ \text { s.t. } & \sum_{e=1}^{N} \mathbf{K}_{e}\left(x_{1 e}, x_{2 e}, \theta_{e}\right) \mathbf{U}=\mathbf{F}, \\ & \theta_{e} \mid \widetilde{z}_{1}=z_{I} \text { and } \widetilde{z}_{2}=z_{I I}, \\ & x_{1 e}, x_{2 e} \mid \sigma_{e, I} \leq 0 \text { and } \sigma_{e, I I} \leq 0, \\ & \sum_{e=1}^{N} \mathbf{U}_{e}^{T} \mathbf{K}_{e}\left(x_{1 e}, x_{2 e}, \theta_{e}\right) \mathbf{U}_{e} \leq \alpha \mathcal{C}_{0}, \\ & x_{\min } \leq x_{1 e}, x_{2 e} \leq 1 .\end{cases}
$$

The minimization unknowns of the above statements are the sets $x_{1 e}$ and $x_{2 e}$, i.e. the element-wise constant material densities along the symmetry axes of the composite, $\widetilde{z}_{1}$ and $\widetilde{z}_{2}$ respectively. The objective function is especially conceived to evaluate the weight of the optimal design in each one of the sub-regions $\Omega_{1}, \Omega_{2}$ and $\Omega_{3}$. It is recalled that under biaxial compression one has $x_{1 e}=x_{2 e}=1$, whereas $x_{i e}=1$ and $x_{j e}=x_{\text {min }}$ (with $i \neq j$ ) stands under uniaxial compression. In a 0-1 optimal design, "black" regions are those where at least one material density per element is equal to one.

Eqn. (6.2) enforces elastic equilibrium in discrete form, accounting for the equivalent or- 
thotropic material hold by Eqns. (3-4). Eqn. (6.3) calls for the set of element-wise constant parameters $\theta_{e}$ that provide the same orientation for the symmetry axes of the composite $\widetilde{z}_{1}$ and $\widetilde{z}_{2}$ and the principal stress directions $z_{I}$ and $z_{I I}$, see Section 2. Eqns. (6.4) is needed to enforce a compression-only stress regime in each finite element that models the region made of plain concrete. Any fixed rebar cage can be taken into account by simply neglecting this constraint over the relevant non-design region. Eqn. (6.5) enforces the compliance constraint, depending on the user-defined parameter $\alpha$ and the value $\mathcal{C}_{0}$ that is found for the full domain made of virgin material $\left(x_{1 e}=x_{2 e}=1\right.$ everywhere).

Section 4 provides comparisons in terms of optimal strut-and-tie models achieved through the conventional formulation for linear elastic isotropic material with equal behavior in tension and compression and the proposed approach accounting for concrete as a compression-only material. Eqns. (5) and (6) are preferred to their minimum compliance counterparts to address layouts having the same strain energy, being achieved for the same value of $\alpha$.

\subsection{Problem implementation}

As detailed in Section 1, the statements in Eqns. (5) and (6) are solved through the gradientbased minimizer MMA [38], calling for the computation of the sensitivity at each iteration of the minimization algorithm. The initial guess is the full material domain, that means $x_{e}=1$ in each element in case of isotropic material and $x_{1 e}=x_{2 e}=1$ for the equivalent composite. Solution of Eqn. (5) is conventional, see e.g. [15], whereas some additional handling is operated on the statement in Eqn. (6) to speed up the computation.

At each iteration, a structural analysis is performed according to Eqn. (6.2) for the current values of the minimization unknowns $x_{1 e}$ and $x_{2 e}$, along with the orientations $\theta_{e}$. The principal stress directions $z_{I}$ and $z_{I I}$ are computed and each parameter $\theta_{e}$ is updated in order to provide alignment of the symmetry axes of the composite $\widetilde{z}_{1}$ and $\widetilde{z}_{2}$ with respect to $z_{I}$ and $z_{I I}$, see Eqn. (6.3). Instead of implementing the demanding stress constraints of Eqns. (6.4), the efficient 
penalization approach proposed in [42] is adopted. Accordingly, the penalized densities $\widehat{x}_{1 e}$ and $\widehat{x}_{2 e}$ are introduced. For $i=1,2$ one has:

$$
\begin{cases}\widehat{x}_{i e}=x_{i e}, & \text { if } \widetilde{\sigma}_{e, i} \leq 0 \\ \widehat{x}_{i e}=k x_{i e}, & \text { otherwise }\end{cases}
$$

where $\widetilde{\sigma}_{e, i}$ are the principal stresses in the $e$-th element and $\left.\left.k \in\right] 0,1\right]$ is a penalization parameter that is herein assumed equal to 0.5 when addressing the compression-only concrete. In all the elements accounting for any fixed rebar cage, $k=1$. Eqn. (7) allows for a straightforward computation of a modified compliance $\widehat{\mathcal{C}}$, in which the terms related to any possible positive principal stress are reduced through the parameter $k$ :

$$
\widehat{\mathcal{C}}=\sum_{e=1}^{N} \mathbf{U}_{e}^{T} \mathbf{K}_{e}\left(\widehat{x}_{1 e}, \widehat{x}_{2 e}, \theta_{e}\right) \mathbf{U}_{e}
$$

Providing the MMA with the reduced form of the compliance constraint in Eqn. (6.5) and the relevant sensitivities involving $\partial \widehat{\mathcal{C}} / \partial x_{1 e}$ and $\partial \widehat{\mathcal{C}} / \partial x_{2 e}$, any distribution of stiff material along the weak direction(s) of the compression-only material is prevented. Derivatives of $\mathcal{C}$ with respect to the element-wise constant material densities can be straightforwardly computed through the adjoint method, recalling the constitutive law in Eqns. (3-4).

Four-node Serendipity finite elements are used along with an element-wise constant approximation of the density variables. This discretization provides results that are in full agreement with available analytical and numerical solutions for the equilibrium of two-dimensional no-tension solids. Good accuracy has been found for benchmark examples even in case of coarse meshes, see [37]. When handling isotropic material, this discrete scheme is well-known to be affected by numerical instabilities such as the arising of undesired checkerboard patterns and mesh dependence, see e.g. [30, 43, 44]. The same issues occur with the formulation in Eqn. (6). Many strategies are 
available to solve the aforementioned problems. A density filter approach [45] is herein adopted for both statements in Eqns. (5) and (6) instead of applying the filter to the objective function and its sensitivities, as done in most cases. Focusing on Eqn. (6), the original design variables $x_{i e}$ are transformed in new sets of physical unknowns $\tilde{x}_{i e}$ reading:

$$
\tilde{x}_{i e}=\frac{1}{\sum_{N} H_{e l}} \sum_{N} H_{e l} x_{i l}, \quad H_{e l}=\sum_{N} \max \left(0, r_{\text {min }}-\operatorname{dist}(e, l)\right),
$$

for $i=1,2$. In the above equation $\operatorname{dist}(e, l)$ is the distance between the centroid of the $e-$ th and $l$-th element, whereas $r_{\min }>d_{m}$ is the filter radius, being $d_{m}$ the reference size of the finite elements. The assumption $r_{\text {min }}=1.5 d_{m}$ is done for all the simulations presented in Section 4 .

Problems in Eqns. (5) and (6) are non-convex, meaning that local minima can be found instead of the expected global optimum. To overcome this numerical issue, a multi-start strategy could be implemented initializing the minimization algorithm with different starting guesses. Alternatively, local minima could be avoided by adopting the gradient-based minimizer in its global convergent version (GCMMA) [46]: conservative convex separable approximations of the objective function are used in this release, for which the sequence of iteration points converges toward the set of Karush-Kuhn-Tucker points.

It must be finally remarked that the optimal topology can be non-unique, especially in case of non-symmetric domain and boundary conditions, see e.g. [47, 48], or addressing stress constraints and multiple loading, see in particular [49]. Other sources of non-uniqueness can be found looking at the different assumptions that can be operated on the parameters involved in the material law, see $[50]$. 


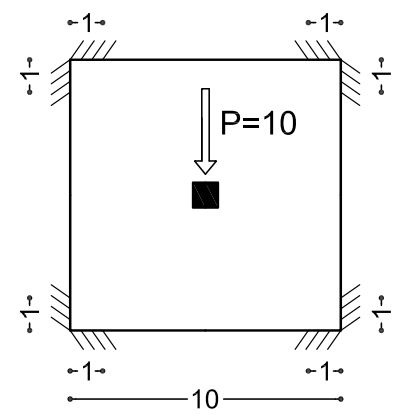

(a)

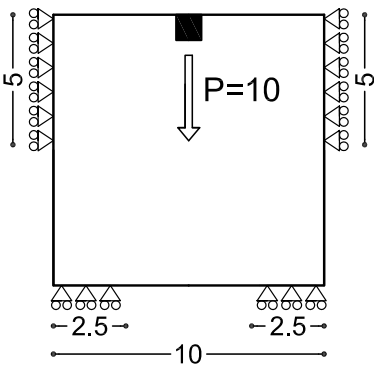

(b)

Figure 2: Geometry and boundary conditions for Examples 1-2 concerning structures made of plain concrete.

\section{Numerical simulations}

This section has the aim of discussing results of the formulation in Eqn. (6), which accounts for concrete as a compression-only material, comparing the achieved strut-and-tie models with optimal layouts found by the conventional formulation in Eqn. (5). The same compliance constraint is used in each comparison. Strut-only models are investigated in Section 4.1 for specimens made of plain concrete, whereas optimal STMs are discussed in Section 4.2 for concrete structures with assigned rebar cages. Specimens of unitary thickness are considered in the numerical simulations, assuming conventionally $E=1 \mathrm{~N} / \mathrm{m}^{2}$ and $\nu=0.3$.

Table 1 shows the number of elements used in the simulations presented next. Fine meshes of bilinear square elements are used to capture the complex strain/stress field arising in the discontinuity regions, while providing the expected accuracy when handling the compliance-constrained optimization.

All the layouts are presented in terms of physical unknowns, that means the set $\tilde{x}_{e}$ for Eqn. (5) and the sets $\tilde{x}_{1 e}$ and $\tilde{x}_{2 e}$ for Eqn. (6). In the latter case, "black" regions are those where at least one material density per element is equal to one, see Section 3.

Tables 2 and 3 compare the optimal STMs in terms of non-dimensional weight (or volume fraction) $\mathcal{W} / \mathcal{W}_{0}$, compliance $\mathcal{C}$ and non-dimensional compliance $\mathcal{C} / \mathcal{C}_{0}$, where subscript 0 refers to 


\begin{tabular}{c|c} 
Example & Elements \\
\hline 1 & 9216 \\
2 & 9216 \\
3 & 4608 \\
4 & 6912 \\
5 & 4608 \\
6 & 9216
\end{tabular}

Table 1: Examples 1-6. Number of elements in the discretization.
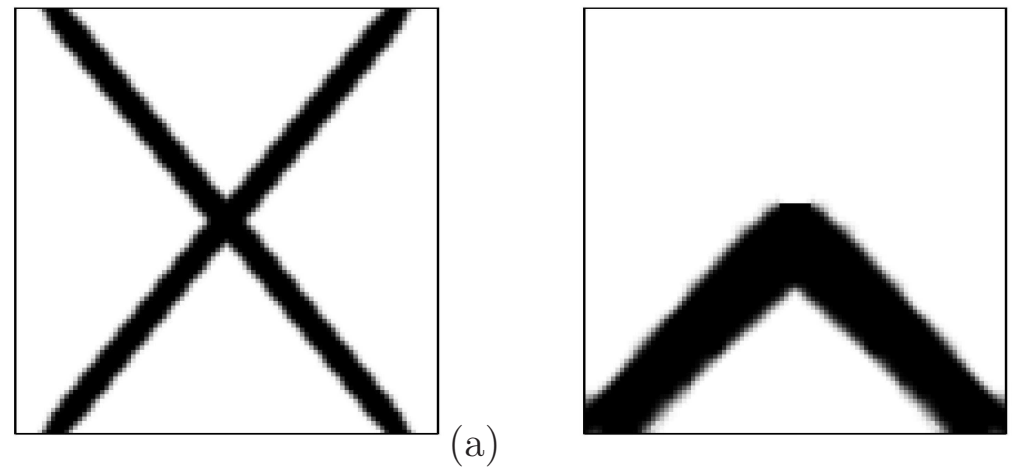

(b)

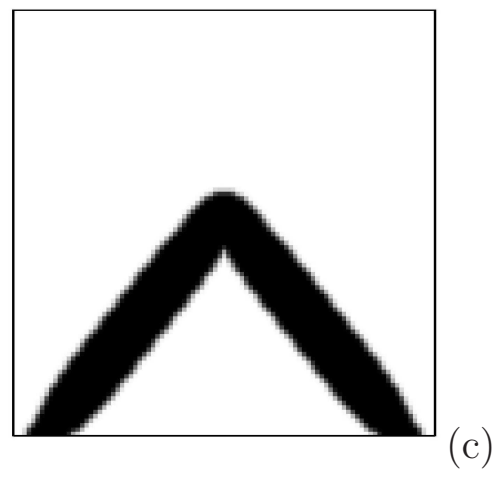

Figure 3: Example 1. Optimal design in case of linear elastic material with equal behavior in tension and compression (a), compression-only material (b) and conventional material with non-active upper constraints (c).

the weight and compliance of the full domain (virgin material).

\subsection{Plain concrete specimens}

\subsubsection{Example 1. The two-bar/four-bar truss example}

Let consider the square domain shown in Figure 2(a), which is loaded by a vertical force applied in the center. Ground supports are available at each corner.

Figure 3(a) shows the optimal solution that is found by the conventional formulation in Eqn. (5), distributing linear elastic isotropic material with equal behavior in tension and compression. A compliance constraint with $\alpha=3$ is enforced, achieving an optimal design whose strain energy is three times that of the full domain of material carrying the same load. A symmetric four-bar 


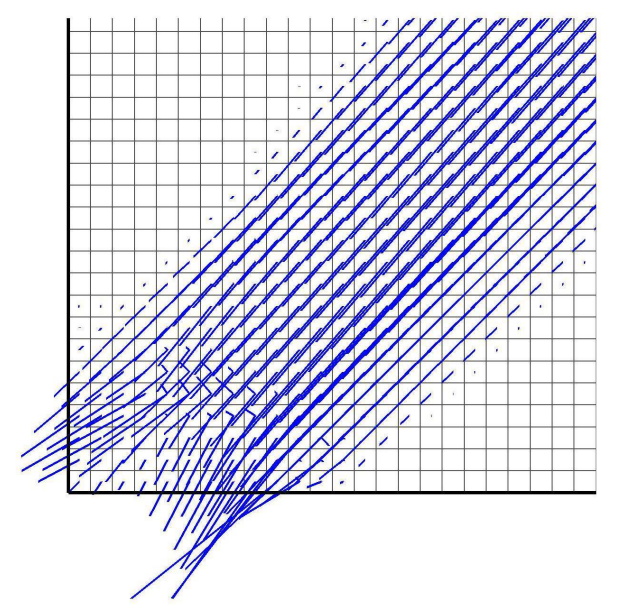

(a)

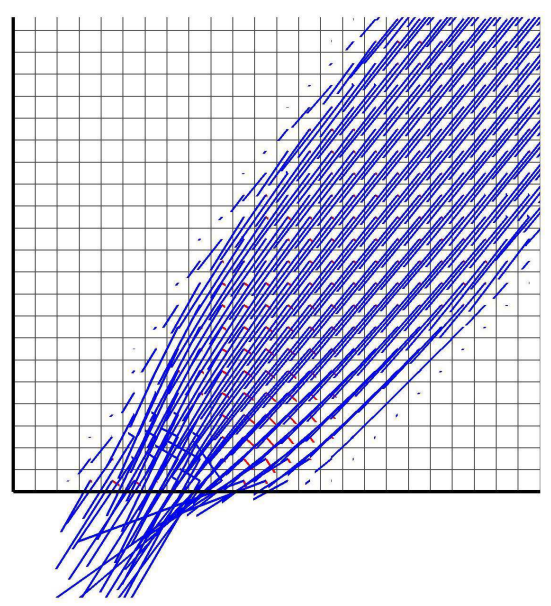

(b)

Figure 4: Example 1. Principal stresses in the lower left strut of the optimal design in case of no-compression material (a) and conventional material with non-active upper constraints (b). Blue lines stands for compression whereas red ones for tension.

truss arises with ties and struts sharing the same structural section. The required volume fraction is around $16 \%$.

The formulation in Eqn. (6) is adopted to investigate the optimal design in case of compressiononly material, having the aim of deriving a strut-only load path for a plain concrete specimen enforcing the same constraint on the strain energy, that means $\alpha=3$. The solution of this benchmark problem consists of a layout made of two struts that connect the load to the lower supports, see Figure 3(b). The required volume fraction increases to approximately $21 \%$.

Finally, Figure 3(c) shows the optimal design in case of conventional material removing the ground constraints at the upper side of the specimen. The achieved solution resembles that of Figure 3(b), but some remarkable differences arise. The strut-only model derived for the compressiononly concrete is made of members inclined of 45 degrees with respect to the horizonal axis. Each strut exploits both horizontal and vertical side of the corner to achieve a fully no-tension connection to the ground. On the contrary, the struts of the model achieved by the conventional formulation in Eqn. (5) follow the shortest path. They connect the load to the inner horizontal 


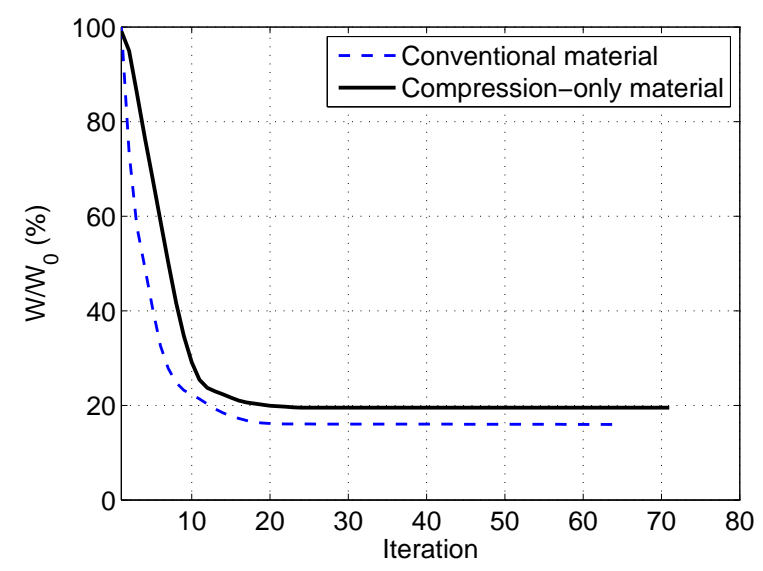

Figure 5: Example 1. Convergence plot for the optimal layouts in Figure 3(b) and Figure 3(c).

\begin{tabular}{c|c|c|c|c|c} 
Ex. & Fig. & Material & $\mathcal{W} / \mathcal{W}_{0}$ & $\mathcal{C}$ & $\mathcal{C} / \mathcal{C}_{0}$ \\
\hline 1 & $3(\mathrm{a})$ & Conventional & 0.160 & 297.48 & 3.0 \\
1 & $3(\mathrm{~b})$ & Compression-only & 0.208 & 297.48 & 3.0 \\
1 & $3(\mathrm{c})$ & Conventional & 0.162 & 297.48 & 3.0 \\
\hline 2 & $6(\mathrm{~b})$ & Conventional & 0.211 & 420.21 & 2.0 \\
2 & $6(\mathrm{a})$ & Compression-only & 0.320 & 420.21 & 2.0
\end{tabular}

Table 2: Examples 1-2. Comparison of the optimal layouts in terms of non-dimensional weight $\mathcal{W} / \mathcal{W}$, compliance $\mathcal{C}(\mathrm{Nm})$ and non-dimensional compliance $\mathcal{C} / \mathcal{C}_{0}$. Subscript 0 refers to the full domain (virgin material).

portion of the ground constraints, with an increased inclination of approximately 50 degrees with respect to the horizontal axis.

Minor differences that concern the shape of the struts can be found in the vicinity of the corner, depending on how local stress paths cope with the ground constraints. No tensile stress arises in the compression-only model, see the principal stresses represented in Figure 4(a), whereas both compression (blue lines) and tension (red lines) are found in case of conventional material with equal behavior in tension and compression, see Figure 4(b).

Finally Figure 5 shows history plots of the objective function for the optimization procedures 

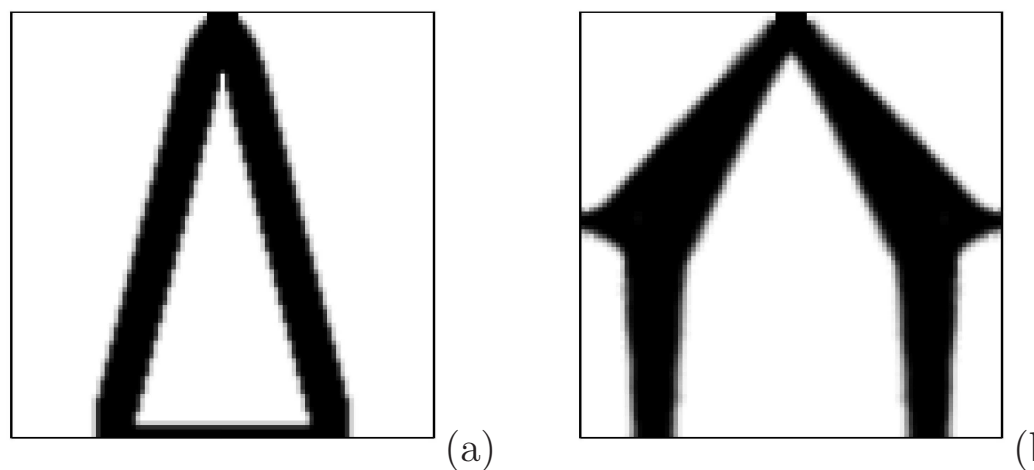

(b)

Figure 6: Example 2. Optimal design in case of linear elastic material with equal behavior in tension and compression (a) and compression-only material (b).

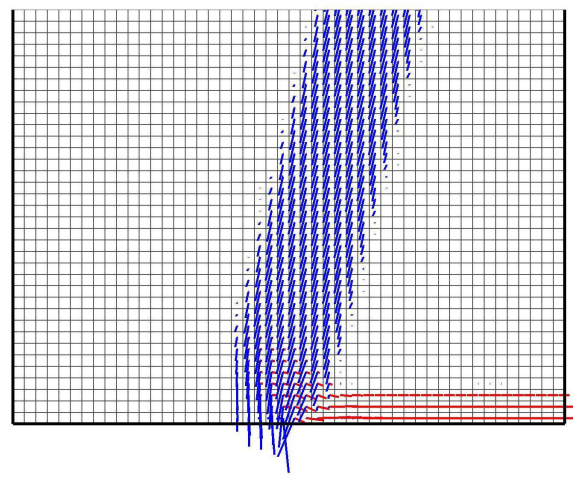

(a)

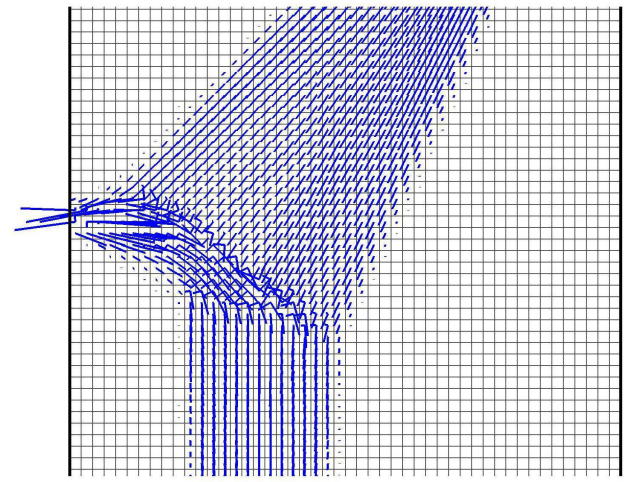

(b)

Figure 7: Example 2. Principal stresses in the elements of the optimal design in case of conventional material (a) and compression-only material (b). Blue lines stands for compression whereas red ones for tension.

achieving the layouts in Figure 3(b) and Figure 3(c). Both simulations refer to the same mesh made of 4608 finite elements. The optimization enforcing the compression-only constraint finds smooth convergence for a slightly increased value of the volume fraction. It calls for some additional steps with respect to the conventional approach.

\subsubsection{Example 2. Anti-funicular load paths in compression-only structures}

The square domain represented in Figure 2(b) is considered, along with a vertical force acting in the middle of the upper side. Constraints against vertical displacements are provided along 


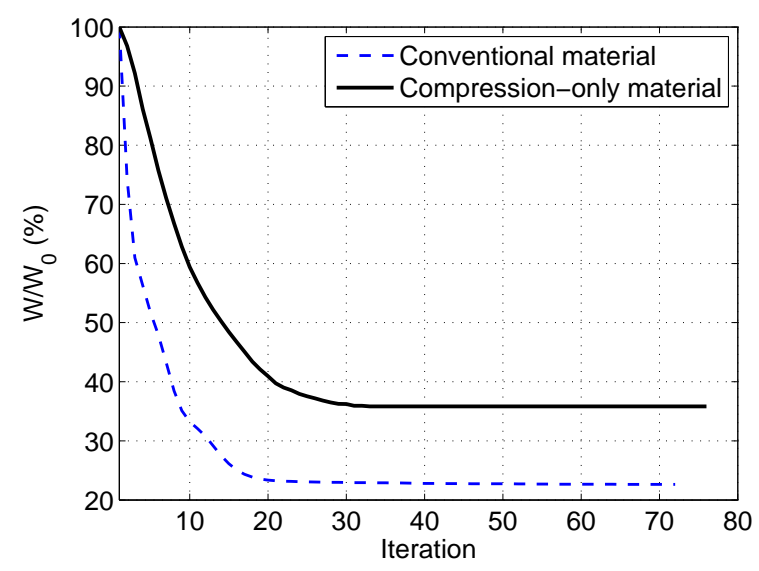

Figure 8: Example 2. Convergence plot for the optimal layouts in Figure 6(a) and Figure 6(b).

the external regions of the lower side, whereas horizontal displacements are constrained along the upper half of each vertical side of the specimen.

Figure 6(a) shows the optimal design in case of conventional material. Enforcing $\alpha=3$, the symmetric behavior in tension and compression leads to a strut-and-tie model that suggests the adoption of a horizontal tensile-stressed reinforcement along the lower side of the specimen, see Figure $7(\mathrm{a})$.

Figure 6(b) shows the optimal layout achieved by the proposed procedure for compressiononly materials for the same value of the parameter $\alpha$. The optimal design takes full advantage of the lateral constraints, which allow steering the inclined struts along the vertical direction. The strut-only load path visualizes the anti-funicular polygon for the applied load, providing details on the connection among its members to preserve equilibrium with no need for any reinforcement, see Figure $7(\mathrm{~b})$.

The above simulations show that the structural behavior of the considered specimen depends remarkably on the assumption concerning the material modeling. According to the strut-and-tie model in Figure 6(a), the more stressed zones of the specimen are those located in the inner region. 

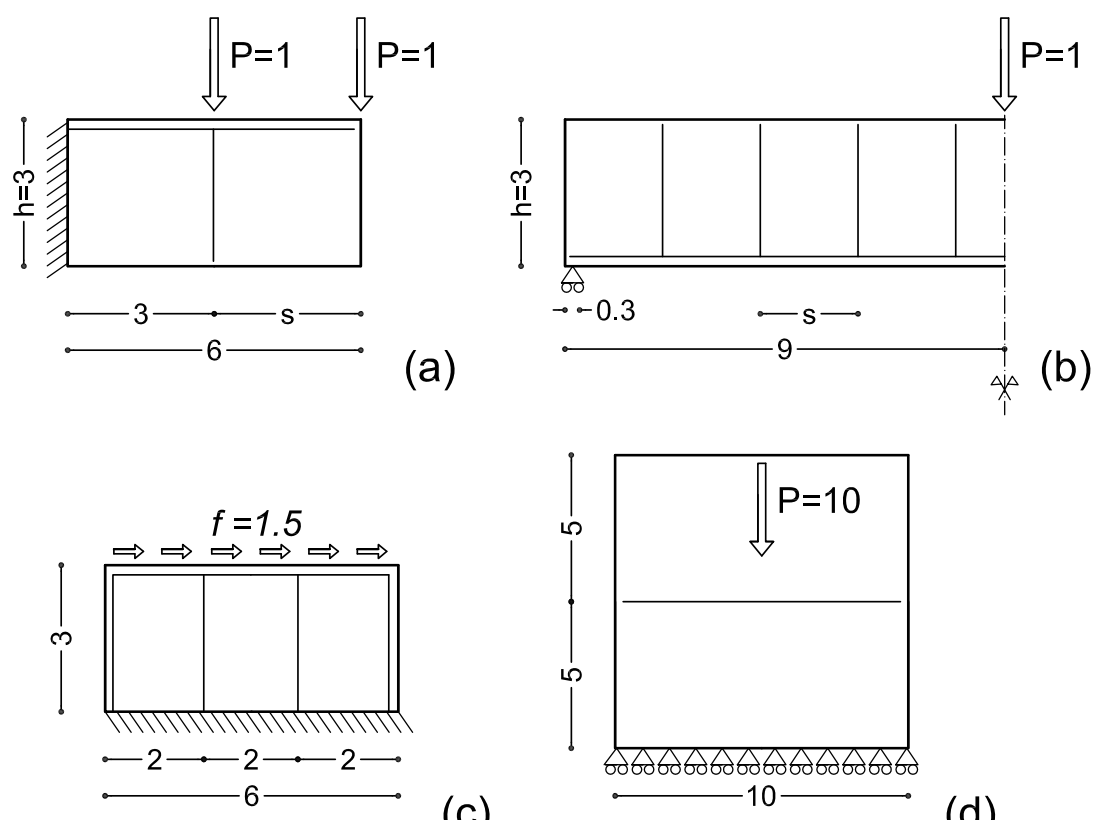

(c)

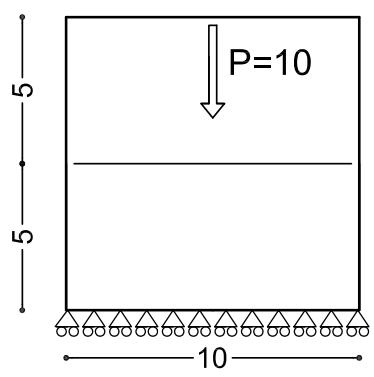

(d)

Figure 9: Geometry, boundary conditions and possible reinforcement for Examples 3-6 concerning structures made of reinforced concrete.

Looking at the strut-only model in Figure 6(b), the outer regions of the specimen are crucial to the equilibrium of the unilateral structural element.

Figure 8 confirms smooth conference of the proposed algorithm, pointing out the increased volume-fraction required by the strut-only-model to preserve the same strain energy stored by the STMs under the same load.

\subsection{Reinforced concrete specimens}

\subsubsection{Example 3. A cantilever beam}

Numerical investigations are performed concerning the cantilever beam represented in Figure 9 (a) and enforcing a compliance constraint with $\alpha=3$.

A first simulation is performed without assigning any fixed region of reinforcement, thus looking for the best STM with no additional manufacturing constraint. Figure 10(a) shows the optimal 

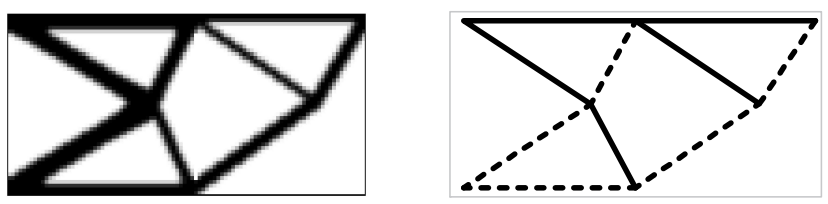

(a)
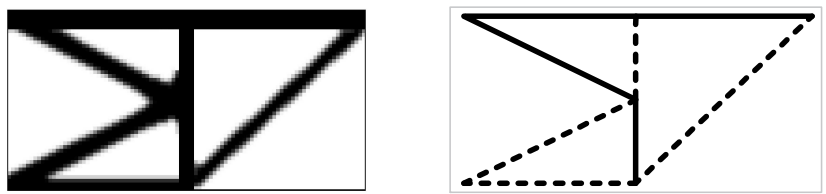

(b)

Figure 10: Example 3. Optimal design and strut-and-tie model in case of linear elastic material with equal behavior in tension and compression: no prescribed reinforcement (a); prescribed longitudinal rebar and stirrup $(s=h)$ (b).
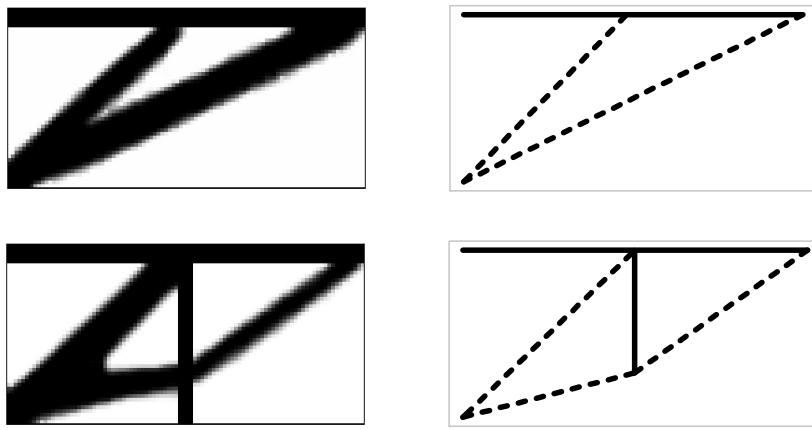

(a)

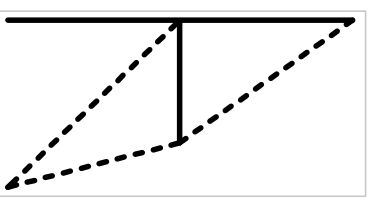

(b)

Figure 11: Example 3. Optimal design and strut-and-tie model in case of compression-only material: prescribed longitudinal rebar (a); prescribed longitudinal rebar and stirrup $(s=h)$ (b). 


\begin{tabular}{c|c|c|c|c|c} 
Ex. & Fig. & Material & $\mathcal{W} / \mathcal{W}_{0}$ & $\mathcal{C}$ & $\mathcal{C} / \mathcal{C}_{0}$ \\
\hline 3 & $10(\mathrm{a})$ & Conventional & 0.287 & 236.02 & 3.0 \\
3 & $10(\mathrm{~b})$ & Conventional $(s=h)$ & 0.322 & 236.02 & 3.0 \\
3 & $11(\mathrm{a})$ & Compression-only & 0.398 & 236.02 & 3.0 \\
3 & $11(\mathrm{~b})$ & Compression-only $(s=h)$ & 0.391 & 236.02 & 3.0 \\
\hline 4 & 12 & Conventional & 0.218 & 122.90 & 4.0 \\
4 & $13(\mathrm{a})$ & Compression-only $(s=3 / 2 h)$ & 0.441 & 122.90 & 4.0 \\
4 & $13(\mathrm{~b})$ & Compression-only $(s=h)$ & 0.415 & 122.90 & 4.0 \\
4 & $13(\mathrm{c})$ & Compression-only $(s=2 / 3 h)$ & 0.404 & 122.90 & 4.0 \\
\hline 5 & 14 & Conventional & 0.326 & 860.61 & 5.0 \\
5 & $15(\mathrm{a})$ & Compression-only & 0.316 & 3442.44 & 20.0 \\
5 & $15(\mathrm{~b})$ & Compression-only & 0.409 & 1721.22 & 10.0 \\
5 & $15(\mathrm{c})$ & Compression-only & 0.518 & 860.61 & 5.0 \\
\hline 6 & 16 & Conventional & 0.284 & 306.18 & 1.5 \\
6 & 17 & Compression-only & 0.394 & 306.18 & 1.5
\end{tabular}

Table 3: Examples 3-6. Comparison of the optimal layouts in terms of non-dimensional weight $\mathcal{W} / \mathcal{W}$, compliance $\mathcal{C}(\mathrm{Nm})$ and non-dimensional compliance $\mathcal{C} / \mathcal{C}_{0}$. Subscript 0 refers to the full domain (virgin material).

design achieved through the conventional formulation in Eqn. (5), along with the strut-and-tie model that can be derived. Inclined compressive paths (dashed lines) connect the loads to the lower corner along the clamped boundary, whereas several bars of reinforcement are needed to steer the stress flux preserving the equilibrium.

An additional simulation is performed to investigate results provided by Eqn. (5) when addressing a specimen with prescribed steel reinforcement. A rebar made of an upper longitudinal bar and a central stirrup is considered, see Figure 9(a). The optimal design is represented in Figure 10(b). Table 3 shows that an increased amount of material is needed to meet the compliance requirement with respect to the solution of Figure 10(a), confirming that the stirrup is not beneficial to the optimal solution being an additional constraint. The achieved STM is not so different with respect to the previous one and an inclined tie arises with respect to the assigned 

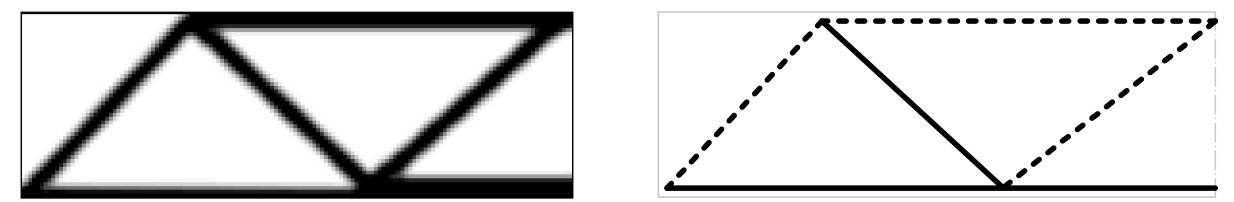

Figure 12: Example 4. Optimal design and strut-and-tie model in case of linear elastic material with equal behavior in tension and compression and no prescribed reinforcement.

reinforcement layout. As expected, the conventional approach for materials with equal behavior in tension and compression can not be used to investigate load paths arising in concrete structures that are reinforced with prescribed rebar cage of any shape.

To this end, the proposed formulation modeling compression-only concrete along with fixed region of conventional material can be successfully implemented. Figures 11 show the optimal layouts and relevant strut-and-tie models provided by Eqn. (6), prescribing an upper horizontal bar only, see Figure 11(a), or the longitudinal rebar coupled with a central stirrup, see Figure 11(b). The same compliance constraint with $\alpha=3$ is considered in both cases. In Figure 11(a), two thick struts connect the loaded points to the lower corner of the clamped side. The equilibrium at the loaded nodes is preserved by the fixed longitudinal steel bar. Focusing on the right load, a strong compressive action arises in the strut, due to its small inclination with respect to the steel bar, approximately 25 degrees. In Figure 11(b), the achieved STM takes full advantage of the central stirrup to create a truss-like structure whose right strut has an increased inclination (approximately 35 degrees) to solve the equilibrium of the loaded node with reduced stresses. Table 3 shows that the achieved design meets the compliance constraint calling for a volume fraction that is lower than the previous design. This confirms the positive contribution due to shear reinforcement and the robustness of the proposed approach exploiting stirrups to find stiff STMs for compression-only materials. 

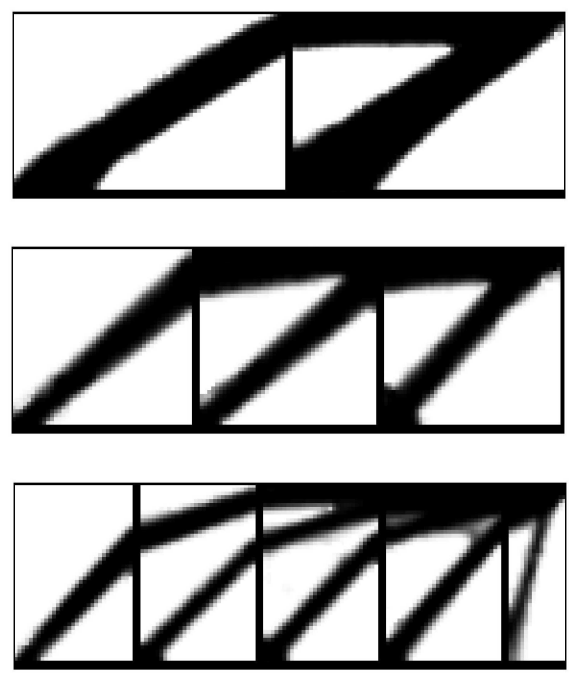

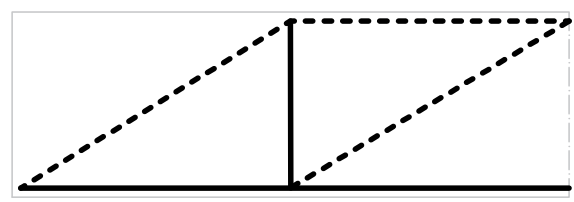

(a)

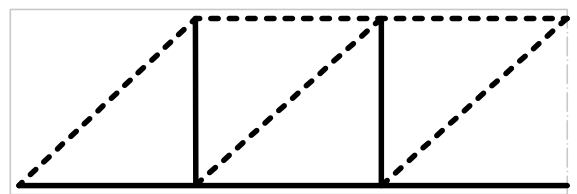

(b)

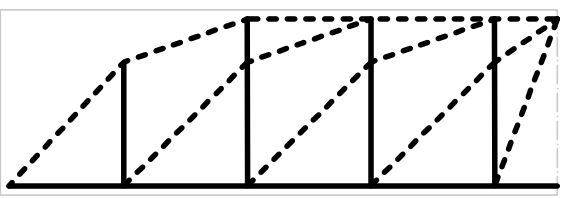

Figure 13: Example 4. Optimal design and strut-and-tie model in case of compression-only material: prescribed longitudinal rebar and stirrup with $s=3 / 2 h(\mathrm{a}) ; s=h(\mathrm{~b}) ; s=2 / 3 h$ (c).

\subsubsection{Example 4. The $M B B$ beam}

Half of the MBB beam depicted in Figure 9(b) is analyzed, due to symmetry reasons, adopting a mesh with 6912 finite elements. At first the formulation in Eqn. (5) is used to investigate an optimal STM for a specimen without any a priori assumption on the reinforcement, enforcing $\alpha=4$. The well-known truss-like structure represented in Figure 12 arises, suggesting the use of struts and ties with an inclination of 45 degrees with respect to the horizontal axis.

The formulation in Eqn. (6) is subsequently used to investigate STMs for the specimen with assigned rebar: a longitudinal reinforcement at the lower side of the domain along with a transversal reinforcement with different stirrup spacing $s$. The same compliance constraint enforcing $\alpha=4$ is adopted. Figures 13(a), (b) and (c) show the optimal layouts and relevant STMs found for $s=3 / 2 h, s=h$ and $s=2 / 3 h$ respectively, being $h$ the height of the specimen, see Figure 9(b).

A set of truss-like structures is achieved that is in full agreement with established models provided by the technical literature on the shear reinforcement of beams, see in particular $[2$, 3]. No tensile-stressed member is found, except for the prescribed longitudinal and transversal 

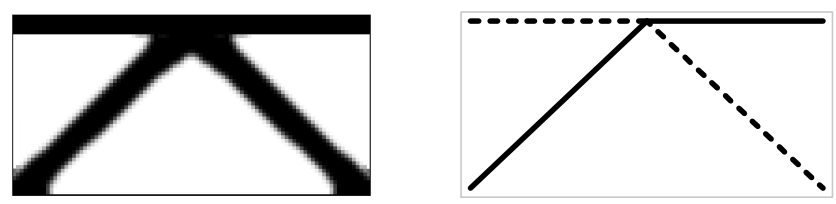

Figure 14: Example 5. Optimal design and strut-and-tie model in case of linear elastic material with equal behavior in tension and compression. A fixed non-design region of reinforcement is assumed at the extrados of the specimen.

reinforcement. Every STM exploits all the available ties, meaning that the number of struts found in the optimal layout strictly depends on the stirrup spacing. For $s=3 / 2 h$ two compressive bars arise, having an inclination of approximately 30 degrees with respect to the horizonal axis. The overall volume fraction is around 44\%. An improved stirrup geometry with $s=h$ allows for three struts with an optimal inclination of 45 degrees. This design achieves a decrease in terms of weight with respect to the previous solution that is around $6 \%$. For $s=2 / 3 h$ the inclination of 45 degrees is preserved for most of the struts found in the lower part of the beam. These struts are deviated by the stirrups to join the horizontal compressive stress path at the extrados of the beam. Three of the four stirrups manage two struts each, meaning that the tensile stress can be non-homogenous along a single transversal bar, for $s<h$. The achieved layout slightly reduces the overall volume fraction with respect to the previous solution, with a decrease that is around $3 \%$.

\subsubsection{Example 5. A shear wall}

This example refers to the shear wall in Figure 9(c). Figure 14 shows the optimal design and strut-and-tie model achieved in case of conventional material with equal behavior in tension and compression, assuming a fixed non-design region of reinforcement along the extrados of the specimen. A compliance constraint enforcing $\alpha=5$ has been adopted to perform this simulation. The same result arises for $\alpha=10$ and $\alpha=20$, as considered next.

The formulation in Eqn. (6) is implemented to investigate the response of the wall, assuming the compression-only behavior for the concrete and considering four vertical bars of reinforcement that are symmetrically distributed along the width. Figure 15(a), (b) and (c) show the optimal 

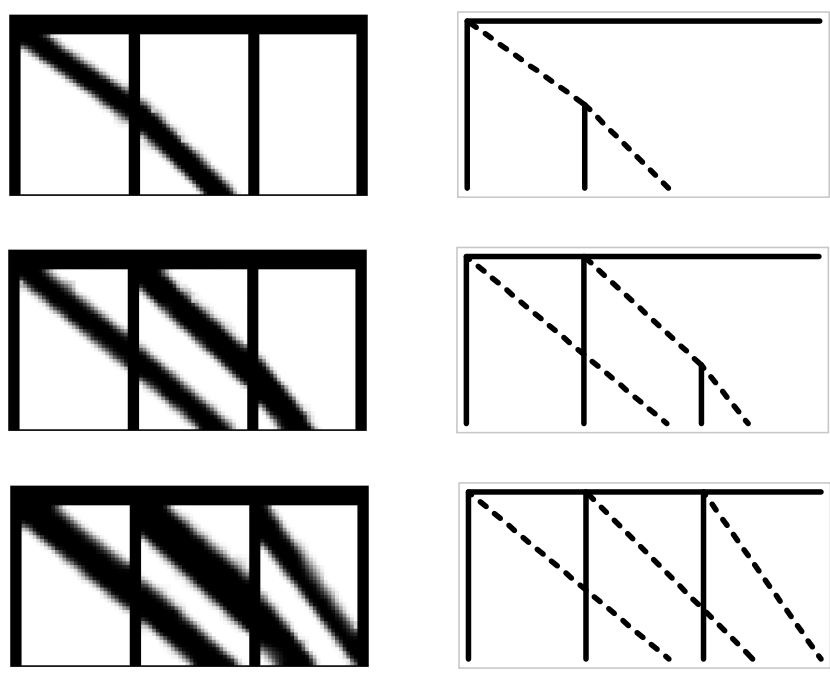

(a)

(b)

(c)

Figure 15: Example 5. Optimal design and strut-and-tie model in case of compression-only material for a prescribed vertical rebar: with $\mathcal{C} / \mathcal{C}_{0}=20(\mathrm{a}) ; \mathcal{C} / \mathcal{C}_{0}=10(\mathrm{~b}) ; \mathcal{C} / \mathcal{C}_{0}=5$ (c).

layouts and relevant STMs found for increasing requirements in terms of allowed strain energy, i.e. $\alpha=20, \alpha=10$ and $\alpha=5$ respectively. In the first case, the whole shear load is carried by the external vertical reinforcement along with a single strut inclined of about 45 degrees with respect to the horizontal axis. A minor deviation of the axis of this element is due to one of the inner bars of the vertical reinforcement. The scheme described above is doubled when the allowed strain energy is halved. The strictest requirement calls for the parallel activation of a third scheme, leading to a third strut whose inclination is ruled by the spacing of the vertical reinforcement.

This example shows that strut-and-tie models can exploit some of the available rebar or the full cage, depending on the strain energy limit enforced through the compliance constraint. If compliant layouts are allowed, the proposed procedure provides load paths involving only elements that are strictly needed to achieve the equilibrium. Requiring an increased stiffness, more articulated STMs can be mobilized. 

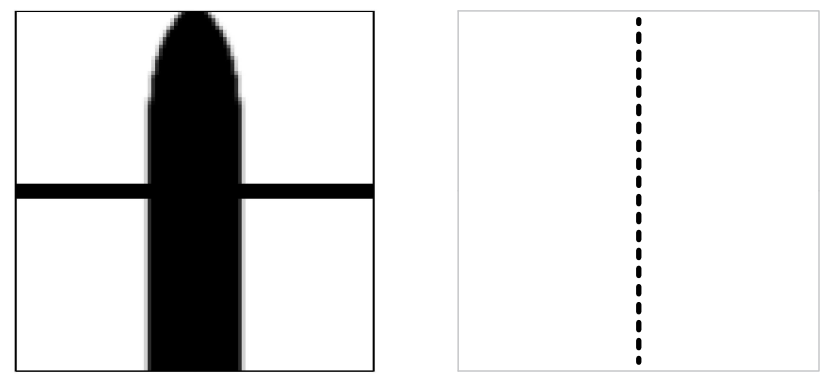

Figure 16: Example 6. Optimal design and strut-and-tie model in case of conventional material with prescribed horizontal reinforcement.
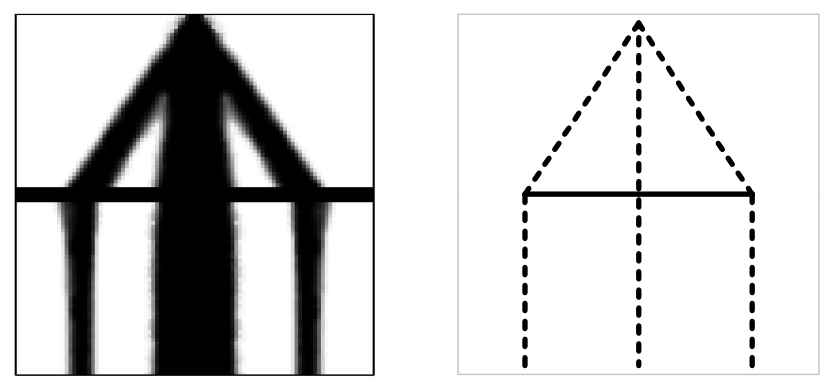

Figure 17: Example 6. Optimal design and strut-and-tie model in case of compression-only material with prescribed horizontal reinforcement. 


\subsubsection{Example 6. Stress diffusion in compression-only concrete}

Conventional formulations for the minimization of the elastic strain energy generate equilibrated truss-like layouts but do not include any explicit enforcement of full consistence of the achieved STM with respect to the stress regime acting in the original structure. Among the others, [7, 27] show that auto-equilibrated compression-only loads may originate strut-only designs, without providing the expected reinforcement that should be placed along the principal tensile directions of the structural element.

To investigate this issue a stress diffusion problem is considered, assuming the geometry and boundary conditions of the specimen reported in Figure 9(d).

A preliminary simulation is performed through Eqn. (5), prescribing a fixed horizontal reinforcement layer at half height of the specimen. As expected, the achieved optimal STM is a single vertical strut connecting the load and the ground constraints. The steel bar is inactive, see Figure 16.

Eqn. (6) is adopted to assess the capability of the proposed approach when copying with the reinforced specimen made of compression-only concrete. Figure 17 shows the optimal design and and the feasible stress paths read through the STM. Part of the load is directly transferred to the ground by the vertical strut. A parallel load paths is additionally needed for the equilibrium of the compression-only medium. Struts inclined of about 30 degrees with respect to the force direction confine the loaded node. They are deviated along the vertical axis by the tensile stresses arising in the prescribed reinforcement bar.

The achieved STM is in full agreement with detailing solutions that are usually exploited in prestressed concrete structures to manage spalling stresses at the anchorages.

It is recalled that strut-and-tie modeling assumes concrete as a compression-only material. The proposed approach succeeds in providing a feasible load path for the considered stress diffusion problem because it effectively enforces this assumption within the optimization procedure. 


\section{Conclusions and perspectives}

A numerical method has been proposed to cope with the energy-based topology optimization of compression-only materials and generate optimal strut-only models for structures made of plain concrete. The approach exploits hyper-elasticity of the considered unilateral material to solve both the inherently non linear equilibrium equation and the topology optimization problem within the same minimization procedure. No handling of the load history is required, thus avoiding demanding incremental procedures for the iterative solution of the state equation.

Numerical investigations addressing plain concrete specimens show that any thick strut arising in the optimal design is robustly managed by the proposed optimization approach. Indeed, the equivalent orthotropic modeling allows for full feasibility of the achieved layouts with respect to the assumption of unilateral behavior of the material. Stress flows are completely free from any tensile component, both in the vicinity of the ground constraints (Example 1) and in the bulk of the elements (Example 2). It must be remarked that feasible load paths are noticeably affected by the compression-only assumption. Strut-only anti-funicular paths replace optimal truss-like layouts found for linear elastic isotropic material with equal behavior in tension and compression. Converge is smooth, whereas the number of iterations is similar to conventional methods.

The proposed numerical approach can be also used to generate optimal strut-and-tie models for concrete structures where fixed regions of reinforcement are prescribed. In full agreement with the STMs theory, the compression-only material that models concrete does not allow for any additional tensile member. Therefore, the arising optimal strut-and-tie models are forced to rely upon the prescribed reinforcement only. Numerical simulations address load paths in case of conventional rebar cages. Both longitudinal and transverse reinforcement are addressed (Example 3), investigating the effect of stirrup spacing in beams (Example 4) and recovering established truss-like models of the technical literature on shear reinforcement. Depending on the strain energy limit that is enforced through the compliance constraint, strut-and-tie models exploit 
some of the available rebar or the full cage (Example 5). In case of stress diffusion problems the achieved optimal load path takes full advantage of any reinforcement for lateral spreading, whereas conventional formulations provide unfeasible strut-only models (Example 6).

The proposed investigations are limited to plane problems. The ongoing research is mainly devoted to the extension of this approach to the three-dimensional framework, having the main goal of investigating stress paths in specimens such as reinforced box-shaped structures or deep beams calling for solid modeling.

[1] Marti P. Basic tools of reinforced concrete beam design. J Am Concr Inst 1985;82(1):46-56.

[2] Ritter W. Die bauweise hennebique. Schweizerische Bauzeitung 1899;33(7):59-61.

[3] Mörsch E. Der Eisenbetonbau, seine Theorie und Anwendung 1908.

[4] Schlaich J, Schaefer K, Jennewein M. Toward a consistent design of structural concrete. PCI J 1987;32(3):74-150.

[5] Ali MA, White RN. Formulation of optimal strut-and-tie models in design of reinforced concrete structures. ACI Special Publications 2000;193:979-998.

[6] Ali MA, White RN. Automatic generation of truss model for optimal design of reinforced concrete structures. ACI Struct J 2001;98(4):431-442.

[7] Biondini F, Bontempi F, Malerba PG. Stress path adapting Strut-and-Tie models in cracked and uncracked R.C. elements. Struct Eng Mech 2001;12(6):685-698.

[8] Biondini F, Bontempi F, Malerba PG. Optimal strut-and-tie models in reinforced concrete structures. Comput Assis Mech Eng Sci 1999;6(3):279-293. 
[9] Liang QQ, Xie YM, Steven GP. Topology optimization of strut-and-tie models in reinforced concrete structures using an evolutionary procedure. ACI Struct J 2000;97(2):322-330.

[10] Xie YM, Steven GP. A simple evolutionary procedure for structural optimization. Comput Struct 1993;49(5):885-896.

[11] Liang QQ, Xie YM, Steven GP. Optimal topology selection of continuum structures with displacement constraints. Comput Struct 2000;77(6):635-644.

[12] Liang QQ, Xie YM, Steven GP. Generating optimal strut-and-tie models in prestressed concrete beams by performance-based optimization. ACI Struct J 2001;98(2):226-232.

[13] Kwak H-, Noh S-. Determination of strut-and-tie models using evolutionary structural optimization. Eng Struct 2006;28(10):1440-1449.

[14] Bendsøe MP, Kikuchi N. Generating optimal topologies in structural design using a homogenization method. Comput Methods Appl Mech Eng 1988;71(2):197-224.

[15] Bruggi M. Generating strut-and-tie patterns for reinforced concrete structures using topology optimization. Comput Struct 2009;87(23-24):1483-1495.

[16] Bruggi M. On the automatic generation of strut and tie patterns under multiple load cases with application to the aseismic design of concrete structures. Adv Struct Eng 2010;13(6):11671181.

[17] Victoria M, Querin OM, Marti P. Generation of strut-and-tie models by topology design using different material properties in tension and compression. Struct Mutltidiscip Opt 2011;44(2):247258. 
[18] Özkal FM, Uysal H. A fully stressed design method to determine the optimum strut-and-tie model for beamcolumn connections. Int J Comput Methods 2012;9(3).

[19] Herranz JP, Maria HS, Gutirrez S, Riddell R. Optimal Strut-and-tie models using full homogenization optimization method. ACI Struct J 2012;109(5):605-613.

[20] Almeida VS, Simonetti HL, Neto LO. Comparative analysis of strut-and-tie models using Smooth Evolutionary Structural Optimization. Eng Struct 2013;56:1665-1675.

[21] Luo Y, Wang MY, Deng Z. Stress-based topology optimization of concrete structures with prestressing reinforcements. Eng Optim 2013;45(11):1349-1364.

[22] Luo Y, Kang Z. Layout design of reinforced concrete structures using two-material topology optimization with Drucker-Prager yield constraints. Struct Multidiscip Opt 2013;47(1):95-110.

[23] Luo Y, Wang MY, Zhou M, Deng Z. Topology optimization of reinforced concrete structures considering control of shrinkage and strength failure. Comput Struct 2015;157:31-41.

[24] Amir O. A topology optimization procedure for reinforced concrete structures. Comput Struct 2013;114-115:46-58.

[25] Amir O, Sigmund O. Reinforcement layout design for concrete structures based on continuum damage and truss topology optimization. Struct Multidiscip Opt 2013;47(2):157-174.

[26] Bogomolny M, Amir O. Conceptual design of reinforced concrete structures using topology optimization with elastoplastic material modeling. Int J Numer Methods Eng 2012;90(13):15781597. 
[27] Gaynor AT, Guest JK, Moen CD. Reinforced concrete force visualization and design using bilinear truss-continuum topology optimization. J Struct Eng 2013;139(4):607-618.

[28] Yang Y, Moen CD, Guest JK. Three-dimensional force flow paths and reinforcement design in concrete via stress-dependent truss-continuum topology optimization. J Eng Mech 2015;141(1).

[29] EN 1992-1-1, 2004. Eurocode 2: Design of concrete structures Part 1-1: General rules and rules for buildings.

[30] Bendsøe MP, Sigmund O. Topology Optimization: Theory, Methods and Applications 2003.

[31] Tjhin TN, Kuchma DA. Integrated analysis and design tool for the strut-and-tie method. Eng Struct 2007;29(11):3042-3052.

[32] Chang CJ, Zheng B, Gea HC (2007) Topology optimization for tension/ compression only design. Proc. of the 7th WCSMO, COEX Seoul, Korea, 2488-2495

[33] Guan H, Steven GP, Xie Y. Evolutionary Structural Optimization incorporating tension and compression materials. Adv Struct Eng 1999;2(4):273-288.

[34] Cai K. A simple approach to find optimal topology of a continuum with tension-only or compression-only material. Struct Multidiscip Opt 2011;43(6):827-835.

[35] Bruggi M, Duysinx P. A stress-based approach to the optimal design of structures with unilateral behavior of material or supports. Struct Multidiscip Opt 2013;48(2):311-326.

[36] Angelillo M, Cardamone L, Fortunato A. A Numerical model for masonry-like structures. J Mech Mater Struct 2010;5(4):583-615. 
[37] Bruggi M. Finite element analysis of no-tension structures as a topology optimization problem. Struct Multidiscip Opt 2014;50(6):957-973.

[38] Svanberg K. Method of moving asymptotes - A new method for structural optimization. Int J Numer Methods Eng 1987;24(2):359-373.

[39] Zhou M, Rozvany GIN. The COC algorithm, Part II: Topological, geometrical and generalized shape optimization. Comput Methods Appl Mech Eng 1991;89(1-3):309-336.

[40] Berke L. An efficient approach to the minimum weight design of deflection limited structures, AFFDL-TM-70-4-FDTR Air Force Flight Dynamics Laboratory, Wright-Patterson AFB 1970.

[41] Berke L. Structural optimization of large structural systems by optimality criteria methods, NASA-TM-105423 National Aeronautics and Space Administration, Washington DC 1992.

[42] Ananiev S. On equivalence between optimality criteria and projected gradient methods with application to topology optimization problem. Multibody Syst Dyn 2005;13(1):25-38.

[43] Sigmund O, Petersson J. Numerical instabilities in topology optimization: A survey on procedures dealing with checkerboards, mesh-dependencies and local minima. Struct Opt 1998;16(1):68-75.

[44] Guest JK, Prévost JH, Belytschko T. Achieving minimum length scale in topology optimization using nodal design variables and projection functions. Int J Numer Methods Eng 2004;61(2):238-254.

[45] Bourdin B. Filters in topology optimization. Int J Numer Methods Eng 2001;50(9):2143-2158. 
[46] Svanberg K. A Class of Globally Convergent Optimization Methods Based on Conservative Convex Separable Approximations. SIAM J Optim 2002;12(2):555-573.

[47] Stolpe M. On some fundamental properties of structural topology optimization problems. Struct Mutltidiscip Opt 2010;41(5):661-670.

[48] Rozvany GIN. On symmetry and non-uniqueness in exact topology optimization. Struct Mutltidiscip Opt 2011;43(3):297-317.

[49] Pintér E, Lengyel A, Lógó J. Structural topology optimization with stress constraint considering loading uncertainties. Period Polytech Civ Eng 2015;59(4):559-565.

[50] Kutylowski R. On nonunique solutions in topology optimization. Struct Mutltidiscip Opt $2002 ; 23(5): 398-403$. 